12

\title{
Прецизионное измерение частот запрещенных переходов с использованием вынужденного комбинационного рассеяния
}

\author{
(C) E.B. Бакланов ${ }^{1,2}$, C.M. Кобцев ${ }^{1}$, А.В. Тайченачев ${ }^{1,2}$ \\ ${ }^{1}$ Институт лазерной физики Сибирского отделения РАН, \\ 630090 Новосибирск, Россия \\ ${ }^{2}$ Новосибирский государственный университет, \\ 630090 Новосибирск, Россия \\ e-mail: baklanov.ev@gmail.com
}

Поступила в редакцию 19.06.2018 г.

Показана возможность прецизионного измерения частот запрещенных переходов холодных атомов в ловушке с помощью вынужденного комбинационного рассеяния (stimulated Raman scattering). Подробно рассмотрен переход $1 S-2 S$ атома водорода, когда атом с уровня $1 S$ переходит на уровень $2 S$ через промежуточный уровень $3 P$. При этом частота поля накачки $(103 \mathrm{~nm})$ близка к частоте перехода $3 P-1 S$, а частота вынужденного рассеяния $(656 \mathrm{~nm})-$ к частоте перехода $3 P-2 S$.

DOI: $10.21883 / \mathrm{OS} .2018 .11 .46823 .177-18$

\section{Введение}

Одним из основных методов лазерной спектроскопии высокого разрешения, который используется для прецизионного измерения частот запрещенных переходов, является метод двухфотонного поглощения без допплеровского уширения [1]. При взаимодействии с атомом двух встречных волн одинаковой частоты устраняются два фактора - допплеровское уширение линии и эффект отдачи при поглощении встречных фотонов. Двухфотонный резонанс обладает важными для спектроскопии свойствами: все атомы независимо от скорости вносят вклад в резонанс, многие запрещенные переходы имеют малую естественную ширину. Основной недостаток этого метода - требуются достаточно большие интенсивности полей, так как их частоты далеко отстроены от резонанса. Однако в настоящее время появились предпосылки для устранения этого недостатка. Методами охлаждения атомов в ловушках получены температуры порядка нанокельвина. Это позволяет считать атомы неподвижными, а при прецизионном измерении частот допплеровское уширение учитывать как малую величину. Эффект отдачи можно оценить с погрешностью, меньшей ошибки измерения, и учесть как поправку при измерении частоты перехода. Поэтому при двухфотонном поглощении частоты встречных волн могут быть выбраны разными и близкими к частотам разрешенных переходов. По существу мы имеем вынужденное комбинационное рассеяние (ВКР).

В этой работе рассмотрена возможность измерения частот запрещенных переходов с помощью ВКР с использованием холодных атомов. Известно, что для $\Lambda$ схем в форме линии вынужденного рассеяния имеется резонанс с однородной шириной перехода между нижними уровнями (ВКР-резонанс), который хорошо исследован теоретически и экспериментально [2]. Фор- мально этот резонанс присутствует в любой задаче с $\Lambda$-схемой, однако наибольший интерес к нему связан с прецизионной спектроскопией и стандартами частоты. Под названием резонанс когерентного пленения населенностей (КПН-резонанс) он используется как репер в компактных коммерческих атомных часах. Для анализа ВКР-резонанса используются стандартные уравнения для матрицы плотности. При температуре охлажденных атомов порядка $1 \mu \mathrm{K}$ можно пренебречь движением атомов и решать эти уравнения как для неподвижного атома. В работе дано решение этих уравнений и получено выражение для ВКР-резонанса.

Точность метода проанализирована на примере запрещенного перехода $1 S-2 S$ атома водорода $(2466 \mathrm{THz})$, который имеет естественную ширину $1.3 \mathrm{~Hz}$. Даны оценки влияния допплеровского уширения, столкновений атомов, полевого сдвига и эффекта отдачи. Показано, что, используя метод ВКР, можно измерить частоту перехода $1 S-2 S$ атома водорода с погрешностью $1 \mathrm{kHz}$. Отметим, что метод ВКР для перехода $1 S-2 S$ по существу является альтернативой методу двухфотонного поглощения без допплеровского уширения. Возможность прецизионного измерения последним методом была рассмотрена в работах [3,4], а эксперименты $[5,6]$ выполнены с относительной погрешностью $\sim 10^{-15}$.

\section{Резонанс в форме линии ВКР}

Уровни трехуровневой схемы будем нумеровать индексами 1, 2, 3 (рисунок). Рассматриваем ВКР, связанное с полем

$$
E(t)=E \exp (-i \omega t)+E^{\prime} \exp \left(-i \omega^{\prime} t\right)+\text { c.c. },
$$

при котором атом с уровня 1 переходит на уровень 2 через промежуточный уровень 3. Рассматривается резонансное приближение, когда частота поля накачки $\omega$ 


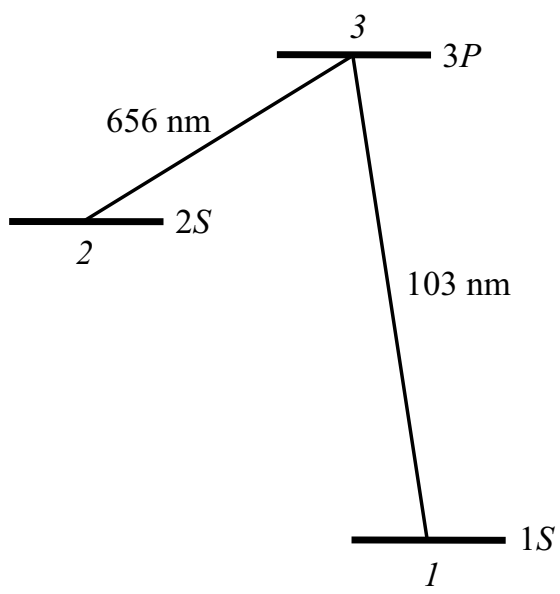

Схема ВКР из основного состояния $1 S$ атома водорода на метастабильный уровень $2 S$ через промежуточный уровень $3 P$.

близка к частоте перехода $\omega_{31}$, а частота вынужденного рассеяния $\omega^{\prime}-$ к частоте перехода $\omega_{32}$. Для элементов матрицы плотности имеем следующие уравнения:

$$
\begin{gathered}
\dot{\rho}_{2}=-V(t) \rho_{23}-V^{*}(t) \rho_{32}, \\
\dot{\rho}_{1}=\gamma \rho_{3}-U(t) \rho_{13}-U^{*}(t) \rho_{31}, \\
\dot{\rho}_{3}+\gamma \rho_{3}=U(t) \rho_{13}+U^{*}(t) \rho_{31}+V(t) \rho_{23}+V^{*}(t) \rho_{32}, \\
\left.\dot{\rho}_{31}+\Gamma .+i \omega_{31}\right) \rho_{31}=U(t)\left(\rho_{1}-\rho_{3}\right)+V(t) \rho_{21}, \\
\dot{\rho}_{32}+\left(\Gamma+i \omega_{32}\right) \rho_{32}=V(t)\left(\rho_{2}-\rho_{3}\right)+U(t) \rho_{12}, \\
\dot{\rho}_{21}+\left(\Gamma_{21}+i \omega_{21}\right) \rho_{21}=-V^{*}(t) \rho_{31}-\rho_{23} U(t) .
\end{gathered}
$$

Здесь $\rho_{i k}=\rho_{k i}^{*}, \rho_{i}=\rho_{i i}, \Gamma_{21}$ - ширина запрещенного перехода $2 \rightarrow 1, \Gamma=\gamma / 2, \gamma-$ радиационная вероятность распада уровня $3 P$,

$$
\begin{gathered}
U(t)=U \exp (-i \omega t), \quad U=d_{31} E / 2 i \hbar, \\
V(t)=V \exp \left(-i \omega^{\prime} t\right), \quad V=d_{32} E^{\prime} / 2 i \hbar,
\end{gathered}
$$

$d_{31}$ и $d_{32}$ - матричные элементы проекций оператора дипольного момента на направление поля. Введем новые переменные:

$$
\begin{gathered}
\rho_{31}=r_{31} \exp (-i \omega t), \quad \rho_{32}=r_{32} \exp \left(-i \omega^{\prime} t\right), \\
\rho_{21}=r_{21} \exp \left(-i \omega t+i \omega^{\prime} t\right) .
\end{gathered}
$$

Учитывая условие $\rho_{0}+\rho_{1}+\rho_{2}=1$ и считая, что $\rho_{2} \ll \rho_{3}$, получим

$$
\begin{gathered}
\dot{\rho}_{2}=-2 \operatorname{Re}\left(r_{32} V^{*}\right), \\
\dot{\rho}_{3}+\gamma \rho_{3}=2 \operatorname{Re}\left(r_{31} U^{*}\right)+2 \operatorname{Re}\left(r_{32} V^{*}\right), \\
\dot{r}_{31}+(\Gamma-i \delta) r_{31}=U\left(1-2 \rho_{3}\right)+V r_{21}, \\
\dot{r}_{32}+\left(\Gamma-i \delta^{\prime}\right) r_{32}=-V \rho_{3}+U r_{21}^{*}, \\
\dot{r}_{21}+\left(\Gamma_{21}-i \Omega\right) r_{21}=-V^{*} r_{31}-U r_{32}^{*} .
\end{gathered}
$$

Здесь $\delta=\omega-\omega_{31}, \quad \delta^{\prime}=\omega^{\prime}-\omega_{32}, \quad \Omega=\omega-\omega^{\prime}-\omega_{21}$. Очевидно, что величина $\dot{\rho}_{2}$ есть вероятность перехода с уровня 1 на уровень 2 под действием двух полей. Обозначив $\dot{\rho}_{2}$ через $W(1 \rightarrow 2)$, перепишем первое уравнение (3):

$$
W(1 \rightarrow 2)=-2 \operatorname{Re}\left(r_{32} V^{*}\right) .
$$

Оставшиеся уравнения (3) служат для определения $r_{32}$. При их решении поля считаются слабыми, удовлетворяющие условиям

$$
|U| / \Gamma \ll 1, \quad|V| / \Gamma \ll 11
$$

В этом случае производными в (3) можно пренебречь и для нахождения $r_{02}$ решать систему следующих уравнений:

$$
\begin{gathered}
\Gamma \rho_{3}=\operatorname{Re}\left(r_{31} U^{*}\right)+\operatorname{Re}\left(r_{32} V^{*}\right), \\
(\Gamma-i \delta) r_{31}=V r_{21}-2 U \rho_{3}+U, \\
\left(\Gamma-i \delta^{\prime}\right) r_{32}=-V \rho_{3}+U r_{21}^{*}, \\
\left(\Gamma_{21}-i \Omega\right) r_{21}=-V^{*} r_{31}-U r_{32}^{*} .
\end{gathered}
$$

Подставим $r_{02}$ из третьего уравнения системы (6) в формулу (4), а в оставшихся уравнениях (6) пренебрежем членами низшего порядка по полю. Это дает

$$
\begin{gathered}
W(1 \rightarrow 2)=\operatorname{Re}\left\{\frac{\left(2|V|^{2} \rho_{3}\right.}{\Gamma-i \delta^{\prime}}\right\}-\operatorname{Re}\left\{\frac{2 U V^{*} r_{21}}{\Gamma-i \delta^{\prime}}\right\}, \\
r_{21}=-\frac{V^{*} U}{(\Gamma-i \delta)\left(\Gamma_{21}-i \Omega\right)}, \\
\rho_{3}=\frac{q}{2} \frac{\Gamma^{2}}{\Gamma^{2}+\delta^{2}} .
\end{gathered}
$$

В результате для вероятности перехода атома из состояния 1 в состояние 2 под действием поля двух частот получим выражение

$$
\begin{gathered}
W(1 \rightarrow 2)=A \frac{\Gamma^{5}}{\left(\Gamma^{2}+\delta^{\prime 2}\right)\left(\Gamma^{2}+\delta^{2}\right)} \\
+A \operatorname{Re}\left\{\frac{\Gamma^{4}}{\left(\Gamma-i \delta^{\prime}\right)(\Gamma-i \delta)\left(\Gamma_{21}-i \Omega\right)}\right\}, \\
A=2 a_{1} a_{2},
\end{gathered}
$$

где величины

$$
a_{1}=|U|^{2} / \Gamma^{2}, \quad a_{2}=|V|^{2} / \Gamma^{2}
$$

являются безразмерными параметрами насыщения на переходах $3 \rightarrow 1$ и $3 \rightarrow 2$ соответственно. Вероятность двухфотонного перехода состоит из двух членов, которые имеют разную физическую природу. Первый член состоит из двух ступенчатых переходов - поглощение фотона с созданием заселенности на верхнем уровне 3, а затем однофотонное излучение. Второй член - ВКР (когерентное поглощение и испускание фотонов), в форме линии которого имеется резонанс с однородной 
шириной запрещенного перехода. При условиях $|\delta| \ll \Gamma$ и $\left|\delta^{\prime}\right| \gg \Gamma$ имеем

$$
W(1 \rightarrow 2)=A\left(\Gamma+\frac{\Gamma_{21} \Gamma^{2}}{\Omega^{2}+\Gamma_{21}^{2}}\right) .
$$

В нашем случае $\Gamma \gg \Gamma_{21}$, поэтому

$$
\begin{gathered}
W(1 \rightarrow 2)=W \frac{\Gamma_{21}^{2}}{\left(\omega-\omega^{\prime}-\omega_{21}\right)^{2}+\Gamma_{21}^{2}}, \\
W=2 a_{1} a_{2} \Gamma^{2} / \Gamma_{21} .
\end{gathered}
$$

Таким образом, имеем резонанс в форме линии ВКР, когда разность частот $\omega-\omega^{\prime}$ равняется частоте перехода $\omega_{21}$.

\section{Точность измерения}

Точность метода проанализируем на примере запрещенного перехода $1 S-2 S$ атома водорода $(2466 \mathrm{THz})$. Уровням $1 S, 2 S, 3 P$ соответствуют номера $1,2,3$ (рисунок), частоты переходов $3 P-1 S(\lambda=103 \mathrm{~nm})$ и $3 P-2 S$ $\left(\lambda^{\prime}=656 \mathrm{~nm}\right)$ есть $\omega_{31}$ и $\omega_{32}$ соответственно. Вероятность перехода в центре резонанса $W$ содержит два малых параметра $a_{1}$ и $a_{2}$, которые, согласно Приложению, равны

$$
a_{1}=I_{1} / I_{31}, \quad a_{2}=I_{2} / I_{32},
$$

где $I_{1}$ и $I_{2}-$ интенсивности на переходах $3 P \rightarrow 1 S$ и $3 P \rightarrow 2 S ; I_{31}=10 \mathrm{~W} / \mathrm{cm}^{2}, I_{32}=0.25 \mathrm{~W} / \mathrm{cm}^{2}$. Радиационная ширина обоих переходов равна $\Gamma=10^{8} \mathrm{~s}^{-1}[8]$. Для того чтобы определить необходимую точность измерения частоты перехода $1 S-2 S$, рассмотрим основные факторы, которые влияют на уширение и сдвиг резонанca (10).

Допплеровское уширение. При измерении частоты перехода $\omega_{21}$ холодные атомы можно считать свободными, а поэтому основным фактором уширения линии является допплеровское уширение. При переходе в формуле (10) к газу атомов надо учесть допплеровское смещение частот $\omega$ и $\omega^{\prime}$ для движущего атома и выполнить усреднение по скоростям с максвелловской функцией распределения. Если волны однонаправленные, то вместо (10) получим

$$
\begin{gathered}
W(1 \rightarrow 2)=W_{D} \exp \left(\left(\omega-\omega^{\prime}-\omega_{21}\right)^{2} / \omega_{D}^{2}\right), \\
W_{D}=2 \sqrt{\pi} a_{1} a_{2} \Gamma^{2} / \omega_{D},
\end{gathered}
$$

где $\omega_{D}=\left(\omega_{31}-\omega_{32}\right) v_{D} / c-$ допплеровская ширина, $v_{D}$ - тепловая скорость. При температуре $T=1 \mu \mathrm{K}$ имеем $\omega_{D}=2 \pi \cdot 10^{6} \mathrm{~s}^{-1}(1 \mathrm{MHz})$. Настройка на центр резонанса с погрешностью $10^{-3} \omega_{D}$ позволяет измерить частоту перехода $\omega_{21}$ с погрешностью порядка $1 \mathrm{kHz}$. В дальнейшем будем ориентироваться на эту точность.

Столкновения атомов. Столкновения атомов водорода в ловушке приводят к сдвигу линии. Измерение этого сдвига выполнено в [7]. Необходимо иметь достаточно низкую плотность атомов (порядка $10^{10} \mathrm{~cm}^{-3}$ ), чтобы при температуре $T=1 \mathrm{~K}$ сдвиг был около $1 \mathrm{kHz}$. Атомы в ловушке занимают область порядка $1 \mathrm{~mm}^{3}$, поэтому их число должно быть не больше $10^{7}$.

Полевой сдвиг. Для оценки зависимости сдвига резонанса от интенсивности поля $a_{2}$ мы решили уравнения (6) с большей точностью, чем в [7]. При $\left|\omega^{\prime}-\omega_{21}\right| \ll \Gamma$ этот сдвиг равен

$$
\Delta \omega_{f}=a_{2}\left(\omega^{\prime}-\omega_{21}\right)
$$

Для интенсивности $I_{2}=2.5 \mathrm{~mW} / \mathrm{cm}^{2}$ имеем $a_{2}=10^{-2}$. При $\omega^{\prime}-\omega_{21}=10^{-2} \Gamma$ получим $\Delta \omega_{f} / 2 \pi=160 \mathrm{~Hz}$.

Эффект отдачи. Сдвиг ВКР-резонанса из-за эффекта отдачи при поглощении и излучении встречных фотонов равен

$$
\Delta \omega=\hbar\left(k^{2}-k^{2}\right) / 2 M,
$$

где $k=2 \pi / \lambda, k^{\prime}=2 \pi / \lambda^{\prime}, M-$ масса атома водорода. Значение этого сдвига $17 \mathrm{MHz}$ вычисляется с погрешностью, гораздо меньшей $1 \mathrm{kHz}$.

Точность регистрации. Пусть $N_{0}$ - число атомов в начальном состоянии $1 S$, а $N-$ в состоянии $2 S$. Так как $N \ll N_{0}$, то можно записать

$$
\partial N / \partial t=W_{D} N_{0}
$$

Подставив $W_{D}$, имеем

$$
\Gamma^{-1} \partial N / \partial t=2 \sqrt{\pi} a_{2} a_{1} N_{0} \Gamma / \omega_{D}
$$

Для измерения атомов в состоянии $2 S$ обычно прикладывается электрическое поле, которое смешивает состояния $2 S$ и $2 P$. Затем регистрируется флуоресценция на лаймановской линии $\lambda=121 \mathrm{~nm}$ (в работе [7] было порядка $10^{5}$ фотонов в секунду, что обеспечивало погрешность регистрации резонанса $1 \mathrm{kHz}$ ). Если считать, что детектируется только десятая часть атомов, то для оценок можно положить $\partial N / \partial t=10^{6} \mathrm{~s}^{-1}$. Учитывая, что $\Gamma=10^{8} \mathrm{~s}^{-1}, \Gamma / \omega_{D}=16, a_{2}=10^{-2}, N_{0}=10^{7}$, получим из соотношения (14) $a_{1}=2 \cdot 10^{-9}$. Так как $I_{1}=a_{1} I_{31}$, To

$$
I_{1}=0.2 \mu \mathrm{W} / \mathrm{cm}^{2}
$$

Размер области атомов в ловушке обычно составляет несколько миллиметров, а поэтому необходимая мощность излучения на длине волны $103 \mathrm{~nm}$ порядка $I_{1} \cdot 10^{-2} \mathrm{~cm}^{2}$, т. е. составляет несколько нановатт.

\section{Заключение}

Показана возможность измерения частот запрещенных перехода с помощью ВКР с использованием холодных атомов. Отметим ряд моментов, которые делают рассмотренный метод перспективным для прецизионной спектроскопии. Атомы при температурах $1 \mu \mathrm{K}$ имеют допплеровскую ширину ВКР-резонанса меньше $1 \mathrm{MHz}$, 
что обеспечивает погрешность измерения частоты перехода меньше $1 \mathrm{kHz}$. Отсутствует квадратичный допплеровский сдвиг, который является источником ошибки в экспериментах с пучками атомов, исключается полевой сдвиг, существенно уменьшаются требуемые интенсивности полей. Необходимые параметры можно выбрать следующим образом (в скобках данные для атома водорода).

1. Задается погрешность измерения $(1 \mathrm{kHz})$.

2. Определяется ширина ВКР-резонанса из возможностей настройки на его центр (1 MHz).

3. По допплеровской ширине резонанса находится температура атомов в ловушке $(1 \mu \mathrm{K})$.

4. Максимально возможное число атомов в ловушке рассчитывается по величине ударного сдвига $\left(10^{7}\right)$.

5. Задается необходимое количество детектируемых атомов в единицу времени $\left(10^{5} \mathrm{~s}^{-1}\right)$.

6. Произведение $a_{2} a_{1}$ находится из соотношения (14). Оно определяет произведение интенсивностей полей.

7. Интенсивности выбираются из условий эксперимента, но так, чтобы был мал полевой сдвиг.

Авторы благодарят Д.В. Бражникова за обсуждение работы и ценные замечания. Работа выполнена при финансовой поддержке гранта РФФИ № 17-02-00292.

\section{Приложение}

Приведенные матричные элементы дипольного момента атома водорода в атомных единицах есть в книге [9] (§ 52):

$$
|\langle 1 S|| d|| 3 P\rangle|^{2}=3^{7} / 2^{13}, \quad|\langle 2 S|| d|| 3 P\rangle|^{2}=2^{20} 3^{7} / 5^{12} .
$$

Квадраты проекций дипольных моментов

$$
\begin{aligned}
& d_{31}^{2}=|\langle 1 S\|d\| 3 P\rangle|^{2} / 3=0.089, \\
& d_{32}^{2}=|\langle 2 S\|d\| 3 P\rangle|^{2} / 3=3.131 .
\end{aligned}
$$

Параметры насыщения $a_{1}=|U|^{2} / \Gamma^{2}, a_{2}=|V|^{2} / \Gamma^{2}$ запишем в виде

$$
a_{1}=I_{1} / I_{31}, \quad a_{2}=I_{2} / I_{32},
$$

где $I_{1}=c|E|^{2} / 8 \pi, I_{2}=c\left|E^{\prime}\right|^{2} / 8 \pi-$ интенсивности на соответствующих переходах,

$$
I_{31}=\frac{q \Gamma^{2}}{2 \pi d_{31}^{2}}, \quad I_{32}=\frac{q \Gamma^{2}}{2 \pi d_{32}^{2}} .
$$

Здесь $I_{31}$ и $I_{32}$ - интенсивности насыщения; $\Gamma=\gamma / 2$, $\gamma=2 \cdot 10^{8} \mathrm{~s}^{-1}$ - радиационная вероятность распада уровня $3 P[8] ; q=c \hbar^{2} /(e a)^{2}, \hbar=10^{-27} \mathrm{ergs}, c=$ $=3 \cdot 10^{10} \mathrm{~cm} \mathrm{~s}^{-1}, e-$ заряд электрона, $a-$ боровский радиус, $(e a)^{2}=6 \cdot 10^{-36} \mathrm{erg} \mathrm{cm}^{3}$. Подставив численные значения, получим $I_{31}=10^{8} \mathrm{erg} / \mathrm{cm}^{2} \mathrm{~s}, \quad I_{32}=$ $=2.5 \cdot 10^{6} \mathrm{erg} / \mathrm{cm}^{2} \mathrm{~s}$. Таким образом имеем

$$
I_{31}=10 \mathrm{~W} / \mathrm{cm}^{2}, \quad I_{32}=0.25 \mathrm{~W} / \mathrm{cm}^{2} .
$$

\section{Список литературы}

[1] Василенко Л.С., Чеботаев В.П., Шишаев А.В. // Письма в ЖЭТФ. 1970. Т. 12. С. 161.

[2] Летохов В.С., Чеботаев В.П. Нелинейная лазерная спектроскопия сверхвысокого разрешения. М.: Наука, 1990. $512 \mathrm{c}$.

[3] Baklanov E.V., Chebotayev V.P. // Opt. Comm. 1974. V. 12. P. 312.

[4] Бакланов Е.В., Чеботаев В.П. // Опт. и спектр. 1975. Т. 38. C. 384.

[5] Niering M., Holzwarth R., Reichert J., Pokasov P., Udem Th., Weitz M., Hänsch T.W., Lemonde P., Santarelli G., Abgrall M., Laurent P., Salomon C., Clairon A. // Phys. Rev. Lett. 2000. V. 84. P. 5496.

[6] Parthey C.G., Matveev A., Alnis J., Bernhardt B., Beyer A., Holzwarth R., Maistrow A., Pohl R., Predehl K., Udem T., Wilken T., Kolachevsky N., Abgrall M., Rovera D., Salomon C., Laurent P., Hänsch T.W. // Phys. Rev. Lett. 2011. V. 107. P. 203001.

[7] Killian T.C., Fried D.G., Willmann L., Landhuis D., Moss S.C., Greytak T.J., Kleppner D. // Phys. Rev. A. 1998. V. 81. P. 3807.

[8] Бете Г., Солпитер Э. Квантовая механика атомов с одним и двумя электронами. М.: Физматлит, 1960. 562 с.

[9] Берестеикий В.Б., Лифиии, Е.М., Питаевский Л.П. Квантовая электродинамика. М.: Физматлит, 2001. 719 с. 